\title{
Correction to: Practical Approaches in M2 Macrophage Biology: Analysis, Pharmacology, and Didactical Interpretation of M2 Macrophage Functions
}

\author{
Correction to: \\ Chapter 10 in: T. Röszer, The M2 Macrophage, \\ Progress in Inflammation Research 86, \\ https://doi.org/10.1007/978-3-030-50480-9_10
}

The original version of chapter 10 in this book was inadvertently published with incomplete captions of Figs. 6 and 7. The figure captions have now been correctedimage courtesy details are added.

The updated online version of the chapter can be found at https://doi.org/10.1007/978-3-030-50480-9_10 